US Army Corps

of Engineers ${ }_{\circledast}$

Engineer Research and

Development Center

\title{
Modernizing Environmental Signature Physics for Target Detection
}

Jay Clausen, R. Affleck, C. Felt, M. Musty, S. Peckham, S.

July 2021

Frankenstein, A. Wagner, and R. Kala, A. Trautz 
The U.S. Army Engineer Research and Development Center (ERDC) solves the nation's toughest engineering and environmental challenges. ERDC develops innovative solutions in civil and military engineering, geospatial sciences, water resources, and environmental sciences for the Army, the Department of Defense, civilian agencies, and our nation's public good. Find out more at www.erdc.usace.army.mil.

To search for other technical reports published by ERDC, visit the ERDC online library at https://erdclibrary.on.worldcat.org/discovery. 


\title{
Modernizing Environmental Signature Physics for Target Detection
}

\author{
Jay Clausen, R. Affleck, C. Felt, M. Musty, S. Peckham, S. Frankenstein, A. Wagner \\ Cold Regions Research and Engineering Laboratory \\ U.S. Army Engineer Research and Development Center \\ 72 Lyme Road \\ Hanover, NH 03755 \\ R. Kala and A. Trautz \\ Geotechnical and Structures Laboratory \\ U.S. Army Engineer Research and Development Center \\ 3909 Halls Ferry Road \\ Vicksburg, MS 39180
}

Final report

Approved for public release; distribution is unlimited.

Prepared for U.S. Army Combat Capabilities Development Command

Fort Belvoir, VA 22060

Under Program Element 0602712A, Project 468349, Task A1010 


\section{Preface}

This study was conducted for the U.S. Army Combat Capabilities Development Command under Army Direct funds, Program Element Number 0602712A, Project Number 468349, and Task Number A1010. The technical monitor was Dr. J ay Clausen.

The work was performed by the Force Projection and Sustainment Branch of the Research and Engineering Division, U.S. Army Engineer Research and Development Center (ERDC), Cold Regions Research and Engineering Laboratory (CRREL). At the time of the presentation, Mr. Justin B. Putnam was Acting Branch Chief; and Mr. J . D. Horne was Division Chief. The Deputy Director of ERDC-CRREL was Mr. David B. Ringelberg, and the Director was Dr. J oseph L. Corriveau.

This material was originally presented at the ERDC RD20 Conference on 29 October 2020 as part of the Battlespace Terrain Mapping Session.

The Commander of ERDC was COL Teresa A. Schlosser and the Director was Dr. David W. Pittman.

DISCLAIMER: The contents of this report are not to be used for advertising, publication, or promotional purposes. Citation of trade names does not constitute an official endorsement or approval of the use of such commercial products. All product names and trademarks cited are the property of their respective owners. The findings of this report are not to be construed as an official Department of the Army position unless so designated by other authorized documents. 


\section{Modernizing Environmental Signature}

Physics for Target Detection

\section{ERDC-CRREL Test Bed}

\section{Partners \\ GSL, CCDC-C5ISR,}

Desert Research Institute



Battlespace Terrain Mapping, Session 211 Approved for public release; distribution unlimited 


\section{OBJECTIVE AND METHODS}

OBJECTIVE: DETERMINE EFFECT OF ENVIRONMENTAL PHENOMONOLOGY ON ABILITY TO DETECT BURIED OBJECTS AND PROVIDE A PREDICTIVE CAPABILITY OF WHEN TARGETS ARE BEST DETECTABLE WITH IR SENSORS

$S W$ \& LWW

Reflectance

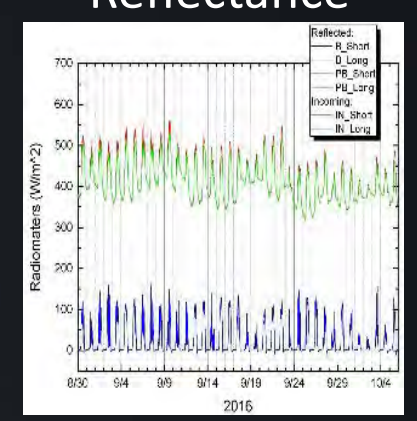

Thermal Flux

Meteorology
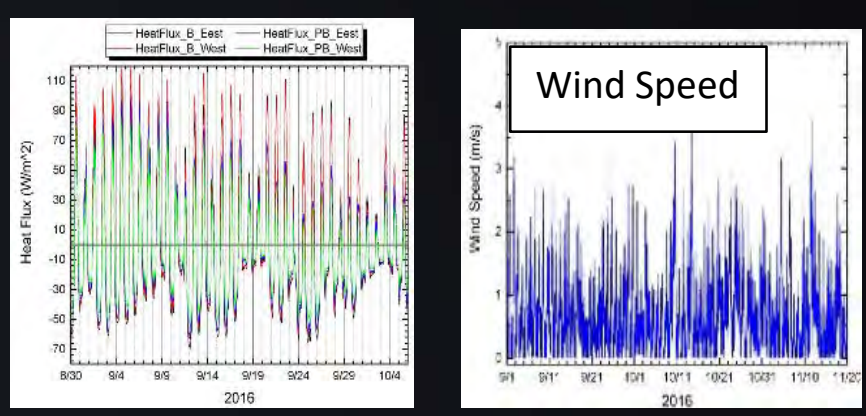

Thermal-Spatia Variability

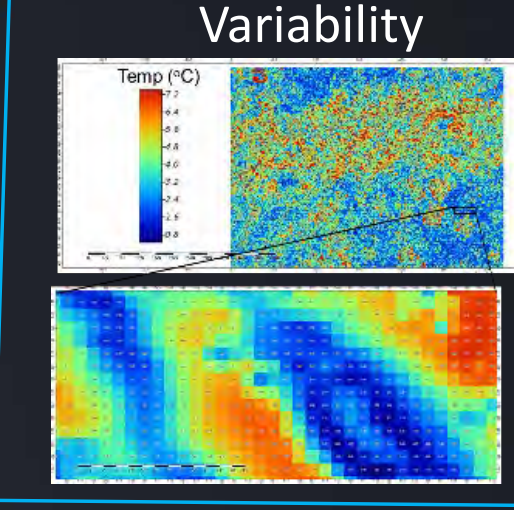

Thermal Discrimination of Materials
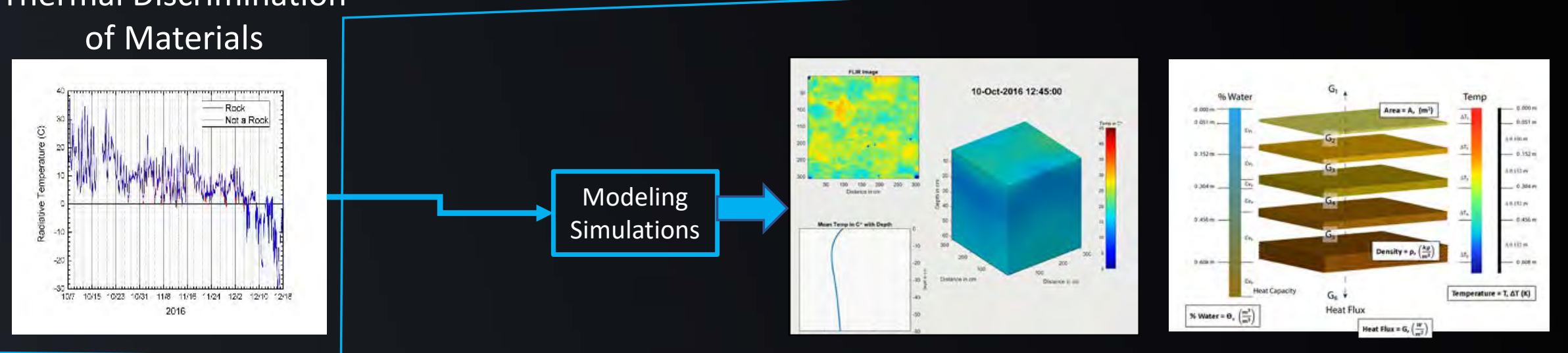


\section{Test Rlot Construction}

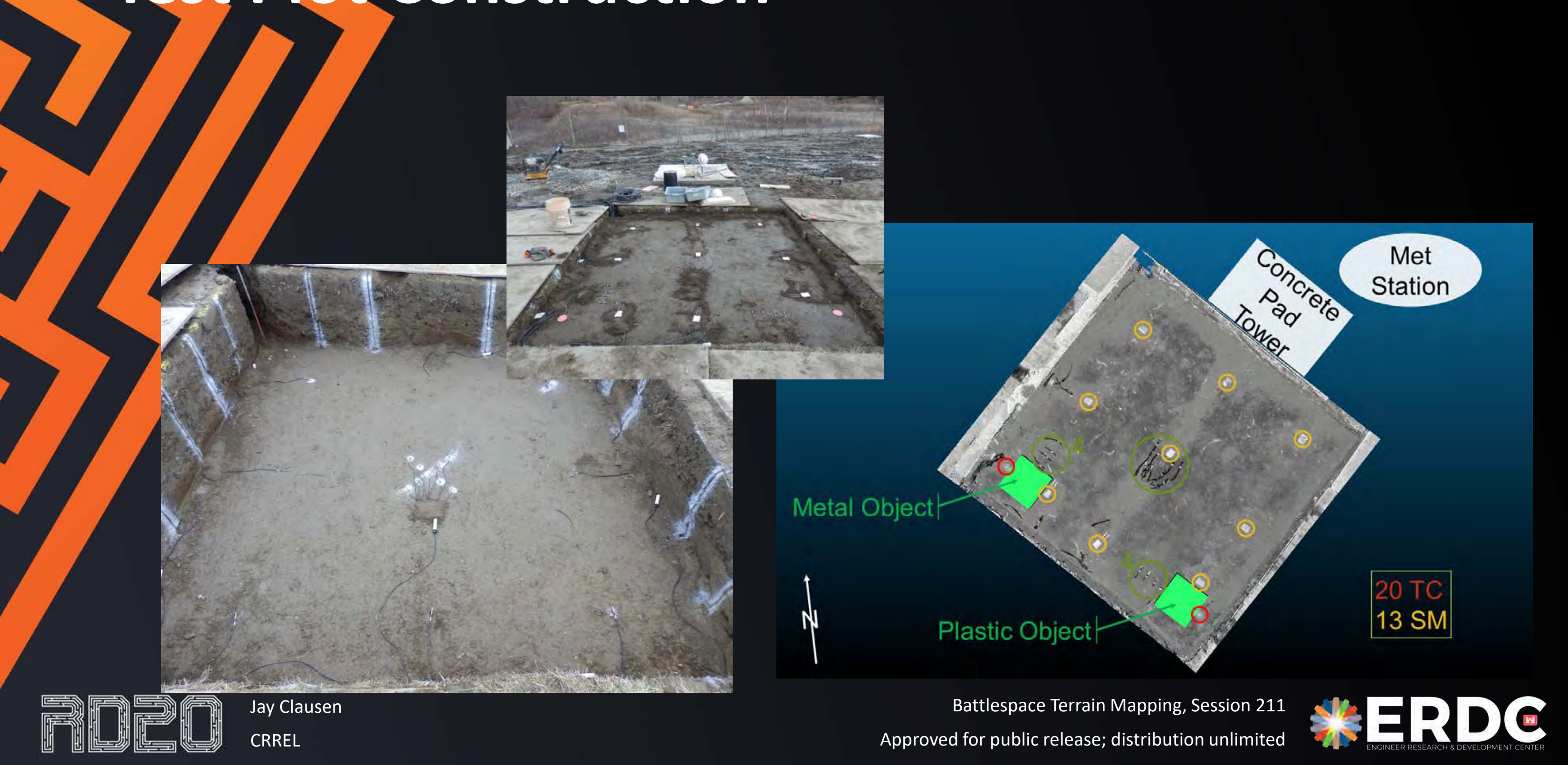




\section{Environmental Measurements}

- Soil Chemistry - ICP-MS, LIBS, XRF, XRD

- Soil reflectance - Field spectrometry

- Surface Topography (roughness) - high resolution LiDAR

- Density - Nuclear Density Gauge

- Temperature (surface and at depth) - Thermocouples, FLIR, PIR

- Heat Flux - Radiometer

- Soil Temperature/Moisture/Conductivity - Field probes

- Grain Size (ASTM)

- Metrological - Air Temperature, Relative Humidity, Precipitation Barometric Pressure, Wind Speed \& Direction

- Solar Radiation (incoming SW \& LW, albedo) - Radiometers 


\section{CRREL Test Plot Features}

- Bare earth with homogenized (disturbed) test bed at CRREL

- Bare earth and vegetated (undisturbed) control areas

- Four buried object locations + surface objects

- FLIR \& EO camera image acquisition

o 15 minute interval over $1 \frac{1}{2}$ years

- Surface air temperature measurements at 4 locations

- Soil parameter measurements

o Thermocouples (150+) at 5 locations at 8 depth intervals

o 26 moisture probes

02 buried heat plates

o 15 minute interval over $1 \frac{1}{2}$ years

- Meteorological parameter measurements

o 5 minute interval over $1 \frac{1}{2}$ years 


\section{Data Sets Available}

\section{- in

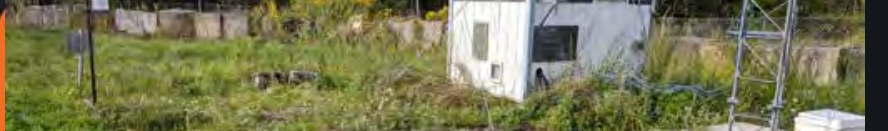

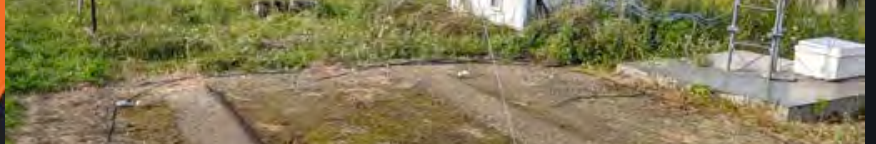

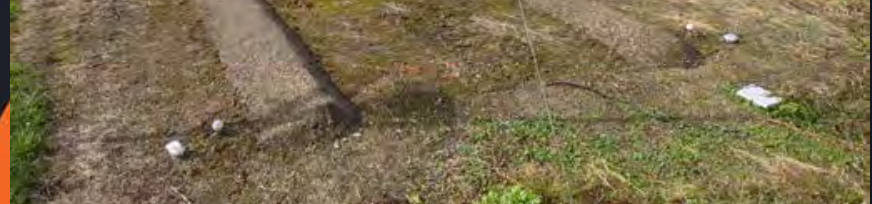

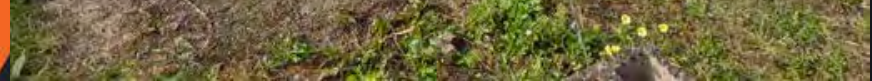
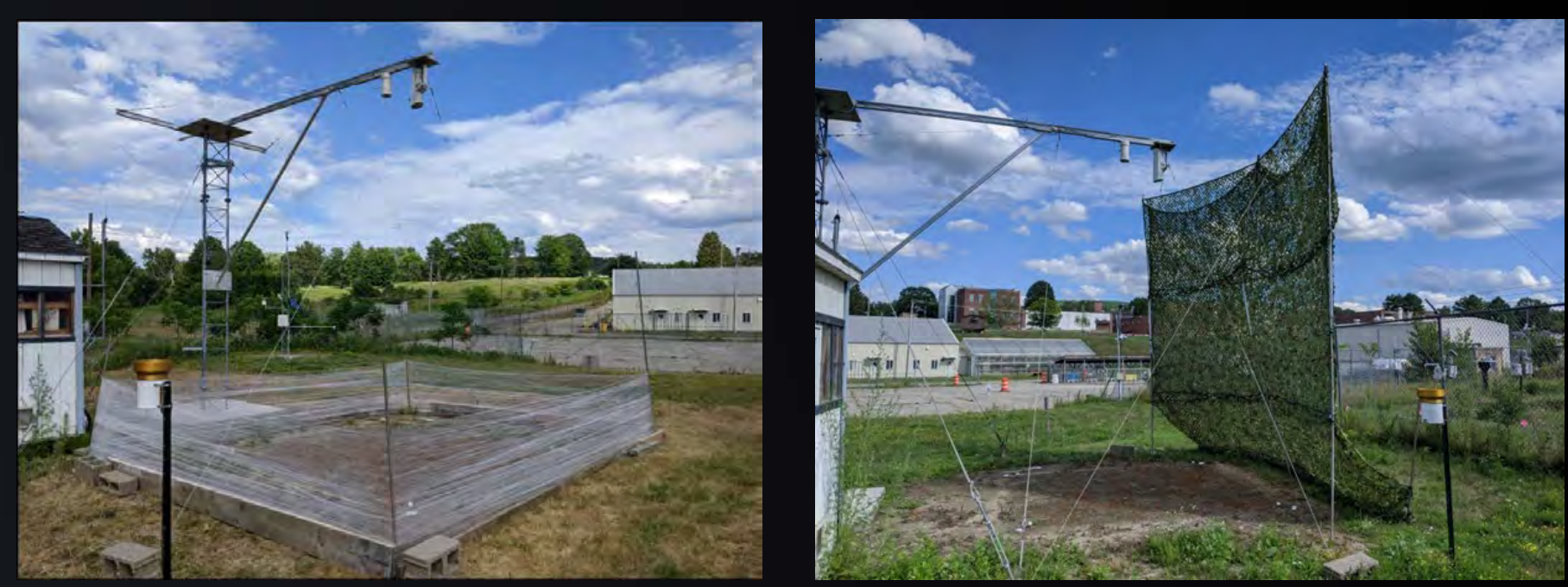

- Phase 1 vegetated on non-vegetated test plots June to November 2016

- Continuous Measurements July to Dec 2018

- Continuous Measurements March to August 2020

- One week study with measurements every 15 seconds

- One week studies each assessing variables: wind speed, solar input, surface topology, soil moisture 
- 3-d animated model simulation (3-months)

o FLIR surface temperatures

o Thermocouple subsurface temperatures

o Soil moisture

o Corresponding incoming short-wave radiation

- Soil thermal heat flow model

- VESPA numerical model test bed 


\section{Technology Transfer}

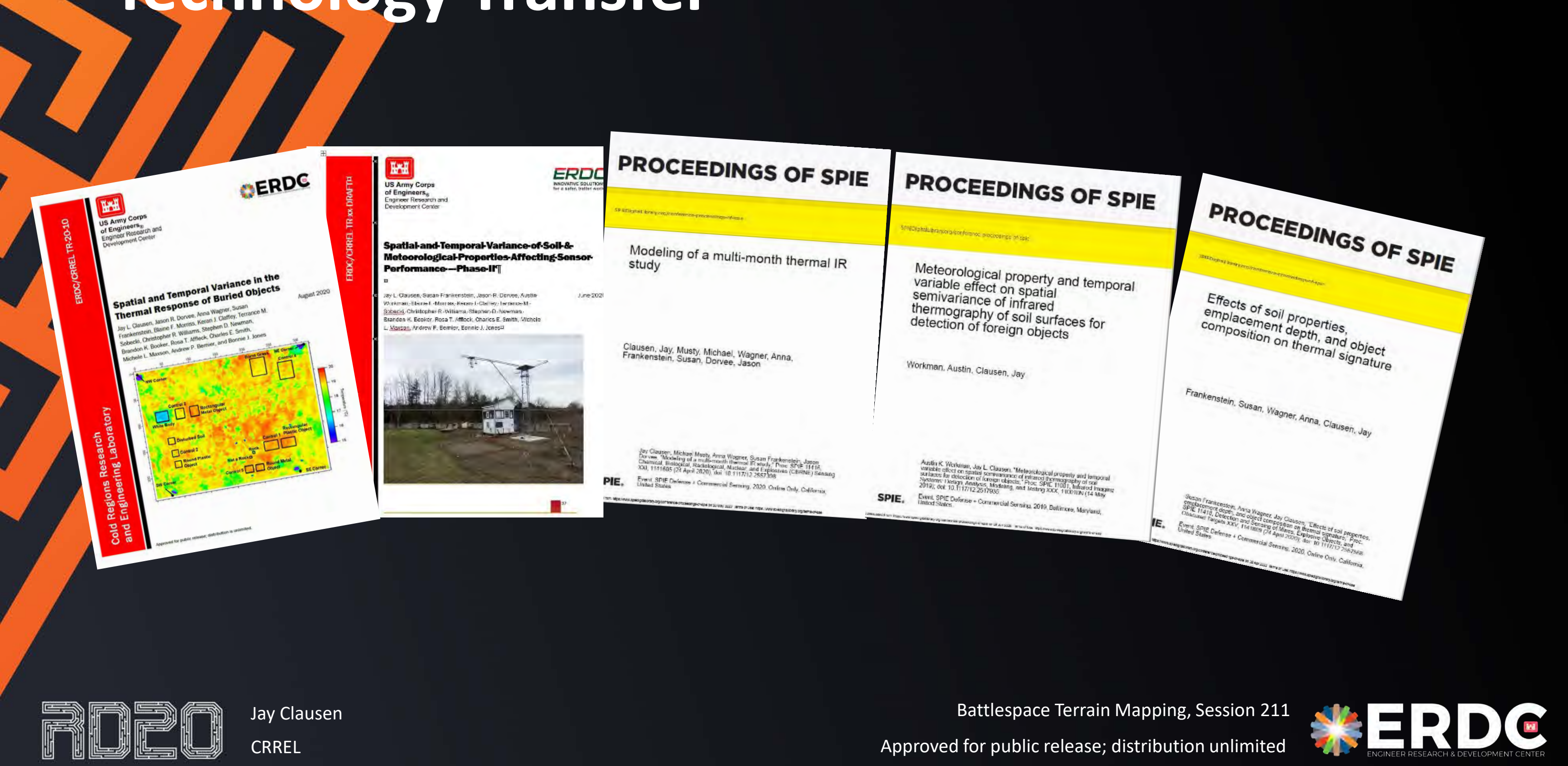




\section{FY21 Tasks}

Task 1 - ML/Al/mage Analysis - Develop statistical learning algorithms to comprehensively analyze and identify important environmental phenomology

Task 2 - CRREL/Test Plot Manipulations - Conduct week long controlled tests forcing single variable (wind,solar radiation, soil moisture, camera angle, surface topology soil density)

Task 3 -Thermal Modeling - Develop a high-fidelity model of the CRREL Test Bed incorporating soil, vegetation, weather, atmosphere and sensor blur/noise inputs to produce realistic synthetic sensor imagery for training object recognition algorithms to improve sensor performance

Task 4 - Thermal Analysis - Develop a heat flow (horizontal and vertical) model to assess buried objects under differing environmental conditions

Task 5 - Multilevel Modeling - Determine quantifiable relational properties between surface radiation and surface soil temperature with and without buried objects

Task 6 - Seasonal Analysis - Assess the seasonal impacts (freeze-thaw, frozen soil, snow) on surface soil temperatures as it relates to thermal IR observations

- Task 7 - Application Development - Explore methods to identify and develop means to geospatially display guidance/recommendations regarding terrain impacts 


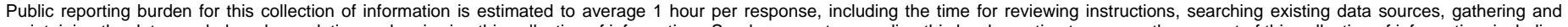



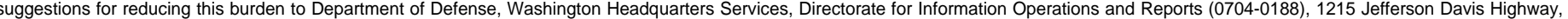

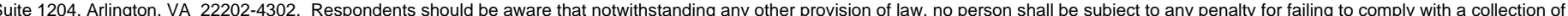
information if it does not display a currently valid OMB control number. PLEASE DO NOT RETURN YOUR FORM TO THE ABOVE ADDRESS.

\section{\begin{tabular}{l|l} 
1. REPORT DATE & 2. REPORT TYPE
\end{tabular} \\ Julv 2021 \\ 4. TITLE AND SUBTITLE}

Modernizing Environmental Signature Physics for Target Detection
3. DATES COVERED (From - To)

FY2018-FY2021

5a. CONTRACT NUMBER

5b. GRANT NUMBER

5c. PROGRAM ELEMENT NUMBER

0602712A

5d. PROJECT NUMBER

468349

5e. TASK NUMBER

A1010

5f. WORK UNIT NUMBER

8. PERFORMING ORGANIZATION REPORT NUMBER

ERDC MP-21-8

Geotechnical and Structures

Laboratory (GSL)

3909 Halls Ferry Road

Vicksburg, MS 39180-6199

72 Lyme Road
Hanover, NH 03755-1290

AND ADDRESS(ES)

10. SPONSOR/MONITOR'S ACRONYM(S)

9. SPONSORING I MONITORING AGENCY NAME(S) AND ADDR
U.S. Army Combat Capabilities Development Command

AMSEL-RD-NV-RM-OPS

10221 Burbeck Road

Fort Belvoir, VA 22060

11. SPONSOR/MONITOR'S REPORT NUMBER(S)

\section{DISTRIBUTION I AVAILABILITY STATEMENT}

Approved for public release; distribution is unlimited.

\section{SUPPLEMENTARY NOTES}

This material was originally presented at the ERDC RD20 Conference on 29 October 2020 as part of the Battlespace Terrain Mapping Session.

\section{ABSTRACT}

The objective of this study was to determine the effect of environmental phenomonology on the ability to detect buried objects and to provide a predictive capability of when targets are best detectable with IR sensors. Jay Clausen presented this material at the ERDC RD20 Conference.

\section{SUBJECT TERMS}

Earth temperature; environmental conditions; infrared detectors; military surveillance

\section{SECURITY CLASSIFICATION OF:}

\section{a. REPORT}

Unclassified

\section{b. ABSTRACT}

Unclassified c. THIS PAGE

Unclassified

\section{LIMITATION} OF ABSTRACT

UU

\section{NUMBER} OF PAGES

15 19a. NAME OF RESPONSIBLE PERSON

19b. TELEPHONE NUMBER (include area code) 